\title{
Quantum gauge boson propagators in the light front
}

\author{
A. T. Suzuki ${ }^{a *}$ and J.H.O. Sales ${ }^{b}$ \\ ${ }^{a}$ Department of Physics, North Carolina State University, \\ Raleigh, NC 27695-8202 \\ ${ }^{b}$ Faculdade de Tecnologia de São Paulo-DEG, \\ Pça. Coronel Fernando Prestes, \\ 01124-060, São Paulo, SP
}

(Dated: October 8, 2018)

\begin{abstract}
Gauge fields in the light front are traditionally addressed via the employment of an algebraic condition $n \cdot A=0$ in the Lagrangian density, where $A_{\mu}$ is the gauge field (Abelian or non-Abelian) and $n^{\mu}$ is the external, light-like, constant vector which defines the gauge proper. However, this condition though necessary is not sufficient to fix the gauge completely; there still remains a residual gauge freedom that must be addressed appropriately. To do this, we need to define the condition $(n \cdot A)(\partial \cdot A)=0$ with $n \cdot A=0=\partial \cdot A$. The implementation of this condition in the theory gives rise to a gauge boson propagator (in momentum space) leading to conspicuous non-local singularities of the type $(k \cdot n)^{-\alpha}$ where $\alpha=1,2$. These singularities must be conveniently treated, and by convenient we mean not only matemathically well-defined but physically sound and meaningfull as well. In calculating such a propagator for one and two noncovariant gauge bosons those singularities demand from the outset the use of a prescription such as the Mandelstam-Leibbrandt (ML) one. We show that the implementation of the ML prescription does not remove certain pathologies associated with zero modes. However we present a causal, singularity-softening prescription and show how to keep causality from being broken without the zero mode nuisance and letting only the propagation of physical degrees of freedom.
\end{abstract}

PACS numbers: 12.39.Ki,14.40.Cs,13.40.Gp

\section{THE LIGHT-FRONT "ENVIRONMENT"}

In 1949 Dirac [1], showed in his pioneering work on relativistic dynamics that it is possible to construct dynamical forms from the description of a initial state of a given relativistic system in any space-time surface whose lengths between points have no causal connection. The dynamical evolution corresponds to the system following a trajectory through the hyper-surfaces. For example, the hyper-surface $t=0$ is our three-dimensional space. It is invariant under translations and rotations. However, in any transformation of inertial reference frames which involves "boosts", the temporal coordinate is modified, and therefore the hyper-surface in $t=0$. Other hyper-surfaces can be invariant under some type of "boost". It is the case of the hyperplane called null plane, defined by $x^{+}=t+z$, which in analogy to the usual coordinate system, is commonly referred to as the "time" coordinate for the front form (light front). For example, a "boost" in the $z$ direction does not modify the null plane.

A point in the usual four-dimensional space-time is defined through the set of coordinates $\left(x^{0}, x^{1}, x^{2}, x^{3}\right)$, where $x^{0}$ is the time coordinate, that is, $x^{0}=t$, with the usual convention of taking the speed of light equal to unity, $c=1$. The other coordinates are the three-dimensional Euclidean space coordinates $x^{1}=x, x^{2}=y$ and $x^{3}=z$.

The light-front coordinates are defined in terms of these by the following relations:

$$
\begin{aligned}
x^{+} & =x^{0}+x^{3}, \\
x^{-} & =x^{0}-x^{3}, \\
\vec{x}^{\perp} & =x^{1} \vec{i}+x^{2} \vec{j},
\end{aligned}
$$

where $\vec{i}$ and $\vec{j}$ are the unit vectors in the direction of the coordinates $x$ and $y$. The null plane is defined by $x^{+}=0$, that is, this condition defines the hyper-surface which is tangent to the light cone, the reason why some authors call those light-cone coordinates.

Note that for the usual four-dimensional Minkowski space-time whose metric $g^{\mu \nu}$ is defined such that its signature is $(1,-1,-1,-1)$ we have

$$
x^{+}=x^{0}+x^{3}=x_{0}-x_{3} \equiv \lambda x_{-},
$$

\footnotetext{
* On sabbatical leave

Permanent address: Instituto de Física Teórica-UNESP, Rua Pamplona 145 - 01405-900 - São Paulo, SP - Brazil.
} 


$$
\begin{aligned}
& x^{-}=x^{0}-x^{3}=x_{0}+x_{3} \equiv \lambda x_{+}, \\
& \vec{x}^{\perp}=x^{1} \vec{i}+x^{2} \vec{j}=-x_{1} \vec{i}-x_{2} \vec{j} \equiv-\vec{x}_{\perp},
\end{aligned}
$$

We emphasize that in the extreme right-hand side of the above two first lines we did not equate $x_{0} \mp x_{3}=x_{\mp}$ but there is a normalizing factor $\lambda=2$ due to the structure of the four-dimensional Minkowski metric translated into the light front coordinates. In other words, $g^{+-}=2$, whereas $g_{+-}=\frac{1}{2}$. We could, of course, have symmetrized them, using, for example:

$$
x^{+}=\frac{x^{0}+x^{3}}{\sqrt{2}}=\frac{x_{0}-x_{3}}{\sqrt{2}} \equiv x_{-},
$$

The initial boundary conditions for the dynamics in the light front are defined in this hyper-plane. Note that the $x^{+}$axis is orthogonal to the plane $x^{+}=0$. Therefore, a displacement of this hyper-surface for $x^{+}>0$ is analogous to the displacement of the plane $t=0$ for $t>0$ of the usual four-dimensional space-time. With this analogy we identify $x^{+}$as the "time" coordinate for the null plane. Of course, since there is a conspicuous discrete symmetry between $x^{+} \leftrightarrow x^{-}$, one could choose $x^{-}$as his "time" coordinate. However, once chosen, one has to stick to the convention adopted. We shall adhere to the former one.

The canonically conjugate momenta for the coordinates $x^{+}, x^{-}$and $x^{\perp}$ are defined respectively by:

$$
\begin{aligned}
& k^{+}=k^{0}+k^{3}, \\
& k^{-}=k^{0}-k^{3}, \\
& k^{\perp}=\left(k^{1}, k^{2}\right) .
\end{aligned}
$$

The scalar product in the light front coordinates becomes therefore

$$
a^{\mu} b_{\mu}=\frac{1}{2}\left(a^{+} b^{-}+a^{-} b^{+}\right)-\vec{a}^{\perp} \cdot \vec{b}^{\perp}
$$

where $\vec{a}^{\perp}$ and $\vec{b}^{\perp}$ are the transverse components of the four vectors. All four vectors, tensors and other entities bearing space-time indices such as Dirac matrices $\gamma^{\mu}$ can be expressed in this new way, using components $(+,-, \perp)$.

From (4) we can get the scalar product $x^{\mu} k_{\mu}$ in the light front coordinates as:

$$
x^{\mu} k_{\mu}=\frac{1}{2}\left(x^{+} k^{-}+x^{-} k^{+}\right)-\vec{x}^{\perp} \cdot \vec{k}^{\perp} .
$$

Here again, in analogy to the usual four-dimensional Minkowski space-time where such a scalar product is

$$
x^{\mu} k_{\mu}=x^{0} k^{0}-\mathbf{x} \cdot \mathbf{k}
$$

where $\mathbf{x}$ is the three-dimensional vector, with the energy $k^{0}$ associated to the time coordinate $x^{0}$, we have the lightfront "energy" $k^{-}$associated to the light-front "time" $x^{+}$. Note, however, that there is a crucial difference between the two formulations: while the usual four-dimensional space-time is Minkowskian, the light-front coordinates project it onto two sectorized two-dimensional Euclidean spaces, namely $(+,-)$, and $(\perp, \perp)$.

In the Minkowski space described by the usual space-time coordinates we have the relation between the rest mass and the energy for the free particle given by $k^{\mu} k_{\mu}=m^{2}$. Using (4), we have

$$
k^{\mu} k_{\mu}=\frac{1}{2}\left(k^{+} k^{-}+k^{-} k^{+}\right)-\vec{k}^{\perp} \cdot \vec{k}^{\perp}
$$

so that

$$
k^{-}=\frac{\vec{k}^{\perp 2}+m^{2}}{k^{+}} .
$$

Note that the energy of a free particle is given by $k^{0}= \pm \sqrt{m^{2}+\mathbf{k}^{\mathbf{2}}}$, which shows us a quadratic dependence of $k^{0}$ with respect to $\mathbf{k}$. These positive/negative energy possibilities for such a relation were the source of much difficulty in the interpretation of the negative energy particle states in the beginning of the quantum field theory descriprion for particles, finally solved by the antiparticle interpretation given by Feynman. In contrast to this, we have a linear dependence between $\left(k^{+}\right)^{-1}$ and $k^{-}$(see Eq.(7)), which immediately reminds us of the non-relativistic quantum mechanical type of relationship for one particle state systems. However, herein one is squarely confronted with the fact that for the light-front "energy" $k^{-}$there is a troublesome feature emerging: The vanishing of the $k^{+}$ momentum component leads to a divergence in the energy relation. This is the famous zero-mode problem in the light front milieu. 


\section{THE CLASSICAL VECTOR GAUGE BOSON PROPAGATOR}

In a recent work of ours [2], we showed that a single Lagrange multiplier defined by $(n \cdot A)(\partial \cdot A)$ with $n \cdot A=\partial \cdot A=0$ at the classical level leads to a propagator in the light-front gauge that has no residual gauge freedom left.

Thus, for the relevant gauge fixing term that enters in the Lagrangian density which we define as

$$
(n \cdot A)(\partial \cdot A)=0
$$

gives for the Abelian gauge field Lagrangian density:

$$
\mathcal{L}=-\frac{1}{4} F_{\mu \nu} F^{\mu \nu}-\frac{1}{2 \alpha}\left(2 n_{\mu} A^{\mu} \partial_{\nu} A^{\nu}\right)=\mathcal{L}_{\mathrm{E}}+\mathcal{L}_{G F}
$$

where the gauge fixing term is conveniently written so as to symmetrize the indices $\mu$ and $\nu$, and the gauge parameter can assume complex values. By partial integration and considering that terms which bear a total derivative don't contribute and that surface terms vanish since $\lim _{x \rightarrow \infty} A^{\mu}(x)=0$, we have

$$
\mathcal{L}_{\mathrm{E}}=\frac{1}{2} A^{\mu}\left(\square g_{\mu \nu}-\partial_{\mu} \partial_{\nu}\right) A^{\nu}
$$

and

$$
\mathcal{L}_{G F}=-\frac{1}{\alpha}(n \cdot A)(\partial \cdot A)=-\frac{1}{2 \alpha} A^{\mu}\left(n_{\mu} \partial_{\nu}+n_{\nu} \partial_{\mu}\right) A^{\nu}
$$

so that

$$
\mathcal{L}=\frac{1}{2} A^{\mu}\left(\square g_{\mu \nu}-\partial_{\mu} \partial_{\nu}-\frac{1}{\alpha}\left(n_{\mu} \partial_{\nu}+n_{\nu} \partial_{\mu}\right)\right) A^{\nu}
$$

To find the gauge field propagator we need to find the inverse of the operator between parenthesis in (12). That differential operator in momentum space is given by $O_{\mu \nu}(k)=-k^{2} g_{\mu \nu}+k_{\mu} k_{\nu}+\frac{1}{\alpha}\left(n_{\mu} k_{\nu}+n_{\nu} k_{\mu}\right)$, so that the propagator of the field, which we call $S^{\mu \nu}(k)$, must satisfy the following equation $O_{\mu \nu} S^{\nu \lambda}(k)=\delta_{\mu}^{\lambda}$, where $S^{\nu \lambda}(k)$ can now be constructed from the most general tensor structure that can be defined, i.e., all the possible linear combinations of the tensor elements that composes it (the most general form includes the light-like vector $m_{\mu}$ dual to the $n_{\mu}[\underline{3}]-$ but for our present purpose it is in fact indifferent):

$$
\begin{aligned}
G^{\mu \nu}(k)= & g^{\mu \nu} A+k^{\mu} k^{\nu} B+k^{\mu} n^{\nu} C+n^{\mu} k^{\nu} D+k^{\mu} m^{\nu} E+ \\
& +m^{\mu} k^{\nu} F+n^{\mu} n^{\nu} G+m^{\mu} m^{\nu} H+n^{\mu} m^{\nu} I+m^{\mu} n^{\nu} J
\end{aligned}
$$

Then, it is a matter of straightforward algebraic manipulation to get the relevant classical propagator in the lightfront gauge, namely,

$$
S^{\mu \nu}(k)=-\frac{1}{k^{2}}\left\{g^{\mu \nu}-\frac{k^{\mu} n^{\nu}+n^{\mu} k^{\nu}}{k^{+}}+\frac{n^{\mu} n^{\nu}}{\left(k^{+}\right)^{2}} k^{2}\right\}
$$

Note that this propagator differs from the traditional two-term light-front propagator in that ours have a third term proportional to $\left(k^{+}\right)^{-2}$, namely

$$
\frac{n^{\mu} n^{\nu}}{\left(k^{+}\right)^{2}} k^{2}
$$

We point out that this exact third term also appears in the canonical quantization of gauge fields in the light-front [4]. Yet, many have been the reasonings and arguments put forth to neglect it altogether. However, we argue back that this term is relevant, first of all from the very fact that such a term ensures there is no anomaly in going from the classical to the quantum propagator and secondly because if $(k \cdot n)^{-1}$ yields a manifestation of zero mode problem, so doubly does the $(k \cdot n)^{-2}$ term, except that they come with opposite signs. We emphasize that no third term such as this one can ever appear at the classical level if one starts off from the Lagrangian density with only one Lagrange multiplier of the form $(n \cdot A)$. 


\section{THE QUANTUM VECTOR GAUGE BOSON PROPAGATOR}

The Feynman quantum propagator for the gauge boson can be derived integrating over all the momenta in (14). Projecting out this propagator on to the light-front we get a gauge boson particle propagating at equal light-front times. We are going to restrict our calculation to the total momentum $P^{+}$positive and corresponding forward light-front time propagation. In this case the propagator from $x^{+}=0$ to $x^{+}>0$ is given by:

$$
\widetilde{S}^{(1) \mu \nu}\left(x_{1}^{\mu}\right)=i \int \frac{d^{4} k_{1}}{(2 \pi)^{4}} \frac{N^{\mu \nu} \mathrm{e}^{-i k_{1}^{\mu} x_{1 \mu}}}{k_{1}^{2}+i \varepsilon}
$$

where

$$
N^{\mu \nu}=\frac{-g^{\mu \nu} k_{1}^{+2}+\left(k_{1}^{\mu} n^{\nu}+n^{\mu} k_{1}^{\nu}\right) k_{1}^{+}-n^{\mu} n^{\nu} k_{1}^{2}}{k_{1}^{+2}} .
$$

Note that because of the structure of the light-front propagator (14) only three of the component projections are non vanishing, namely,

$$
N^{\perp \perp}=-g^{\perp \perp}, \quad N^{\perp-}=\frac{n^{-} k_{1}^{\perp}}{k_{1}^{+}} \quad N^{--}=\frac{n^{-} n^{-} k_{1}^{\perp 2}}{k_{1}^{+2}}
$$

At equal light-front times, we have (we focus on the "energy" integral):

$$
\widetilde{S}^{(1) \mu \nu}\left(x^{+}\right)=\frac{i}{2} \int \frac{d k_{1}^{-}}{(2 \pi)} \frac{N^{\mu \nu} \mathrm{e}^{-\frac{i}{2} k_{1}^{-} x^{+}}}{k_{1}^{+}\left(k_{1}^{-}-\frac{\left(k_{1}^{\perp}\right)^{2}}{k_{1}^{+}}+\frac{i \varepsilon}{k_{1}^{+}}\right)} .
$$

so that, in terms of the component projections we have immediately

$$
\begin{aligned}
& \widetilde{S}^{++}=\widetilde{S}^{+-}=\widetilde{S}^{+\perp}=0 . \\
& \widetilde{S}^{\perp \perp} \neq \widetilde{S}^{\perp-} \neq \widetilde{S}^{--} \neq 0
\end{aligned}
$$

Here the $(\perp, \perp)$ component presents no particular difficulty in evaluation nor does it present any troublesome feature. However, the components $(\perp,-)$ and $(-,-)$, whose relevant computation is technically similar to each other, come with the troublesome feature known as the zero mode problem in the light front. Without loss of generality, we shall therefore restrict ourselves to the analysis of the $(\perp,-)$ component in the following.

In terms of Fourier transform we have (we focus on the "time" variable):

$$
S^{(1) \mu \nu}\left(p^{-}\right)=\int d x^{+} \widetilde{S}^{(1) \mu \nu}\left(x^{+}\right) \mathrm{e}^{\frac{i}{2} p^{-} x^{+}},
$$

so that the component $S^{\perp-}$ is:

$$
\begin{aligned}
S^{(1) \perp-}\left(p^{-}\right) & =i \int \frac{d k_{1}^{-}}{2(2 \pi)} \frac{2(2 \pi) \delta\left(p^{-}-k_{1}^{-}\right) k_{1}^{\perp} n^{-}}{k_{1}^{+}\left(k_{1}^{-}-\frac{\left(k_{1}^{\perp}\right)^{2}}{k_{1}^{+}}+\frac{i \varepsilon}{k_{1}^{+}}\right)}\left[\frac{1}{k_{1}^{+}}\right]_{\mathrm{ML}} \\
& =i \int d k_{1}^{-} \frac{k_{1}^{\perp} n^{-} \delta\left(p^{-}-k_{1}^{-}\right)}{k_{1}^{+}\left(k_{1}^{-}-\frac{\left(k_{1}^{\perp}\right)^{2}}{k_{1}^{+}}+\frac{i \varepsilon}{k_{1}^{+}}\right)}\left[\frac{k_{1}^{-}}{k_{1}^{+}\left(k_{1}^{-}+\frac{i \varepsilon}{k_{1}^{+}}\right)}\right]_{\mathrm{ML}}
\end{aligned}
$$

where

$$
2 \delta\left(p^{-}-k_{1}^{-}\right)=\delta\left(\frac{p^{-}-k_{1}^{-}}{2}\right)=\frac{1}{2 \pi} \int d x^{+} \mathrm{e}^{\frac{i}{2}\left(p^{-}-k_{1}^{-}\right) x^{+}}
$$

and the index ML stands for the Mandelstam-Leibbrandt prescription [5] for the treatment of the $\left(k^{+}\right)^{-1}$ poles, namely,

$$
\left[\frac{1}{k^{+}}\right]_{\mathrm{ML}}=\lim _{\varepsilon \rightarrow 0}\left[\frac{k^{-}}{k^{+} k^{-}+i \varepsilon}\right]_{\mathrm{ML}}
$$


The result is:

$$
S^{(1) \perp-}\left(p^{-}\right)=\frac{\theta\left(p^{+}\right) p^{\perp} n^{-}}{p^{+2}} \frac{i}{\left(p^{-}-K_{0}^{(1)-}+i \varepsilon\right)}
$$

where we have introduced the definition

$$
K_{0}^{(1)-}=\frac{p^{\perp 2}}{p^{+}}
$$

as the light-front Hamiltonian of the free one-particle system. Note that for $x^{+}<0$, the $S^{(1)}\left(x^{+}\right)=0$ because $p^{+}>0$. Moreover, observe that $S^{(1) \perp-}\left(p^{-}\right)$is in an operator form with respect to $p^{+}$and $\vec{p}^{\perp}$. Consequently we have a clear manifestation of the zero mode problem in the factor $\left(p^{+}\right)^{-2}=0$.

We emphasize that even with the use of the Mandelstam-Leibbrandt prescription to handle the non-local singularity, the zero mode problem still lingers on.

\section{THE TWO VECTOR GAUGE BOSON PROPAGATORS}

The two-boson gauge propagator can be derived from the covariant propagator for two particles propagating at equal light-front times. Without losing generality, we are going to restrict our calculation to the total momentum $P^{+}$ positive and corresponding forward light-front time propagation. In this case the propagator from $x^{+}=0$ to $x^{+}>0$ is given by:

$$
\begin{aligned}
\widetilde{S}^{(2) \mu \nu ; \alpha \beta}\left(x^{\prime \mu}, x^{\mu}\right)= & \int \frac{d^{4} k_{1}}{(2 \pi)^{4}} \frac{d^{4} k_{2}}{(2 \pi)^{4}} \frac{i N^{\mu \nu} \mathrm{e}^{-i k_{1}^{\mu}\left(x_{1 \mu}^{\prime}-x_{1 \mu}\right)}}{k_{1}^{2}+i \varepsilon} \\
& \frac{i N^{\alpha \beta} \mathrm{e}^{-i k_{2}^{\mu}\left(x_{2 \mu}^{\prime}-x_{2 \mu}\right)}}{k_{2}^{2}+i \varepsilon} .
\end{aligned}
$$

At equal light-front times $x_{1}^{+}=x_{2}^{+}=0$ and $x_{1}^{\prime+}=x_{2}^{\prime+}=x^{+}$, the propagator is written as:

$$
\widetilde{S}^{(2)}\left(x^{+}\right)=\widetilde{S}_{1}^{(1)}\left(x^{+}\right) \widetilde{S}_{2}^{(1)}\left(x^{+}\right)
$$

where the one-body propagators, $\widetilde{S}_{i}^{(1)}$, corresponding to the light-front propagators of particles $i=1$ or 2 , are defined by Eq.(15). We have explicitly:

$$
\begin{aligned}
\widetilde{S}^{(2) \mu \nu ; \alpha \beta}\left(x^{+}\right) & =i^{2} \int \frac{d k_{1}^{-}}{2(2 \pi)} \frac{d k_{2}^{-}}{2(2 \pi)} \frac{N^{\mu \nu} \mathrm{e}^{-\frac{i}{2} k_{1}^{-} x^{+}}}{k_{1}^{+}\left(k_{1}^{-}-\frac{k_{1}^{\perp 2}-i \varepsilon}{k_{1}^{+}}\right)} \\
& \times \frac{N^{\alpha \beta} \mathrm{e}^{-\frac{i}{2} k_{2}^{-} x^{+}}}{k_{2}^{+}\left(k_{2}^{-}-\frac{k_{2}^{\perp 2}-i \varepsilon}{k_{2}^{+}}\right)} .
\end{aligned}
$$

The Fourier transform to the total light-front energy $P^{-}$is given by

$$
S^{(2) \mu \nu ; \alpha \beta}\left(P^{-}\right)=\int d x^{+} \widetilde{S}^{(2) \mu \nu ; \alpha \beta}\left(x^{+}\right) \mathrm{e}^{\frac{i}{2} P^{-} x^{+}} .
$$

As before, we can recognize immediately that the following components vanish (we ommit the (2) index as well as the $P^{-}$dependence for shortness)

$$
\begin{aligned}
& S^{++,++}=S^{++,+-}=S^{++,+\perp}=S^{++,--}=S^{++,-\perp}=0 \\
& S^{++, \perp \perp}=S^{+-,+-}=S^{+-,+\perp}=S^{+-,--}=S^{+-,-\perp}=0 \\
& S^{+-, \perp \perp}=S^{+\perp,+\perp}=S^{+\perp,--}=S^{+\perp,-\perp}=S^{+\perp, \perp \perp}=0
\end{aligned}
$$

whereas the following ones are the ones that we need to deal with:

$$
\begin{aligned}
& S^{--,--} \neq S^{--,-\perp} \neq S^{--, \perp \perp} \neq 0 \\
& S^{-\perp,-\perp} \neq S^{-\perp, \perp \perp} \neq S^{\perp \perp, \perp \perp} \neq 0 .
\end{aligned}
$$


Let us then evaluate the $(\perp-, \perp-)$-compomnent,

$$
\begin{aligned}
S^{(2) \perp-, \perp-}\left(P^{-}\right) & =-\frac{1}{2(2 \pi)} \int \frac{d k_{1}^{-}}{k_{1}^{+}\left(P^{+}-k_{1}^{+}\right)} \\
& \times\left[\frac{k_{1}^{-}}{k_{1}^{+}\left(k_{1}^{-}+\frac{i \varepsilon}{k_{1}^{+}}\right)}\right]_{\mathrm{ML}} \frac{n^{-} k_{1}^{\perp}}{\left(k_{1}^{-}-\frac{k_{1}^{\perp 2}}{k_{1}^{+}}+\frac{i \varepsilon}{k_{1}^{+}}\right)} \\
& \times\left[\frac{P^{-}-k_{1}^{-}}{\left(P^{+}-k_{1}^{+}\right)\left(P^{-}-k_{1}^{-}+\frac{i \varepsilon}{P^{+}-k_{1}^{+}}\right)}\right]_{\mathrm{ML}} \\
& \times \frac{n^{-}\left(P^{\perp}-k_{1}^{\perp}\right)}{\left(P^{-}-k_{1}^{-}-\frac{\left(P^{\perp}-k_{1}^{\perp}\right)^{2}}{P^{+}-k_{1}^{+}}+\frac{i \varepsilon}{P^{+}-k_{1}^{+}}\right)},
\end{aligned}
$$

where $P^{-,+, \perp}=k_{1}^{-,+, \perp}+k_{2}^{-,+, \perp}$.

We perform the analytical integration in the $k_{1}^{-}$momentum by evaluating the residues at the poles

$$
\begin{aligned}
& k_{1}^{-}=-\frac{i \varepsilon}{k_{1}^{+}} \\
& k_{1}^{-}=P^{-}+\frac{i \varepsilon}{P^{+}-k_{1}^{+}} .
\end{aligned}
$$

It implies that only $k_{1}^{+}$in the interval $0<k_{1}^{+}<P^{+}$gives a nonvanishing contribution to the integration. The result is

$$
S^{(2) \perp-, \perp-}\left(P^{-}\right)=\frac{1}{2} \frac{\theta\left(k_{1}^{+}\right)}{k_{1}^{+2}} \frac{\theta\left(P^{+}-k_{1}^{+}\right)}{\left(P^{+}-k_{1}^{+}\right)^{2}} \frac{i\left(k_{1}^{\perp} n^{-}\right)\left(P^{\perp}-k_{1}^{\perp}\right) n^{-}}{\left(P^{-}-K_{0}^{(2)-}+i \varepsilon\right)},
$$

and just for the purpose of comparison we quote the result for the $(\perp \perp, \perp \perp)$-component,

$$
S^{(2) \perp \perp, \perp \perp}\left(P^{-}\right)=\frac{\theta\left(k_{1}^{+}\right) \theta\left(P^{+}-k_{1}^{+}\right)}{2 k_{1}^{+}\left(P^{+}-k_{1}^{+}\right)} \frac{i\left(-g^{\perp \perp}\right)\left(-g^{\perp \perp}\right)}{\left(P^{-}-K_{0}^{(2)-}+i \varepsilon\right)},
$$

where

$$
K_{0}^{(2)-} \equiv k_{1 \mathrm{on}}^{-}+k_{2 \mathrm{on}}^{-}=\frac{k_{1}^{\perp 2}}{k_{1}^{+}}+\frac{\left(P^{\perp}-k_{1}^{\perp}\right)^{2}}{P^{+}-k_{1}^{+}}
$$

with

$$
k_{i \text { on }}^{-}=\frac{k_{i}^{\perp 2}}{k_{i}^{+}} \quad i=1,2
$$

$K_{0}^{(2)-}$ is the light-front Hamiltonian of the free two-particle system. For $x^{+}<0, S^{(2)}\left(x^{+}\right)=0$ due to our choice of $P^{+}>0$. Observe that $S^{(2)}\left(P^{-}\right)$is written in Eq. (31) and Eq.(32) in operator form with respect to $k_{1}^{+}$and $\vec{k}_{1}^{\perp}$.

We have written down explicitly the result for $S^{(2) \perp \perp, \perp \perp}$ in order to point out that this component is free from the zero mode, but as in the one-boson case before, again we have problems of a divergent factor for $k_{1}^{+}=0$ in (31), which is a manifestation of the zero mode problem lingering on even after the ML prescription has been used. Other non-vanishing components, namely, $S^{(2)--,--}, S^{(2)--,-\perp}, S^{(2)--, \perp \perp}$ and $S^{(2)-\perp, \perp \perp}$ can be evaluated in a similar fashion and all of these also contain zero mode problems.

\section{ZERO MODES THROUGH THE SINGULARITY-SOFTENING PRESCRIPTION}

Zero modes in the light front milieu is a very subtle problem which for years have been challenging us with the best of our efforts to understand it, to make it manageable and to make some physical sense out of it. We have already 
learned that a prescription to handle those singularities cannot be solely mathematically well-defined — that is not enough - we now know that the prescription must be causal, that is, it needs to ascertain that its implementation does not violate causality [ $[$ ]. ML prescription has been heralded as the causal prescription to handle the light-front singularites and many a calculation do confirm that it has solved many difficulties concerning one- and two-loop quantum corrections to Feynman diagrams. However, as seen in the previous sections, ML prescription does not remove the pathological zero modes in the one- and two-vector gauge boson propagation at the quantum level. We therefore come to the place most important in this work: The introduction of a novel prescription that is causal and can handle the light-front singularities which does not leave remnant zero modes is presented and applied to the one and two propagating vector bosons in the light-front gauge.

The index SS stands for this singularity-softening prescription for the treatment of the $\left(k^{+}\right)^{-1}$ poles (cf. [7]), namely,

$$
\begin{aligned}
{\left[\frac{1}{k^{+}}\right]_{\mathrm{SS}} } & =\lim _{\varepsilon \rightarrow 0}\left[\frac{k^{2}}{k^{+}\left(k^{2}+i \varepsilon\right)}\right]_{\mathrm{SS}} \\
& =\lim _{\varepsilon \rightarrow 0}\left[\frac{k^{-}-k_{\mathrm{on}}^{-}}{k^{+}\left(k^{-}-k_{\mathrm{on}}^{-}+\frac{i \varepsilon}{k^{+}}\right)}\right]_{\mathrm{SS}}
\end{aligned}
$$

\section{THE ONE GAUGE BOSON CASE}

The component $S^{\perp-}$ is:

$$
\begin{aligned}
S^{(1) \perp-}\left(p^{-}\right)= & i \int d k_{1}^{-} \frac{k_{1}^{\perp} n^{-} \delta\left(p^{-}-k_{1}^{-}\right)}{k_{1}^{+}\left(k_{1}^{-}-\frac{k_{1}^{\perp 2}}{k_{1}^{+}}+\frac{i \varepsilon}{k_{1}^{+}}\right)}\left[\frac{1}{k_{1}^{+}}\right]_{\mathrm{SS}} \\
= & i \int d k_{1}^{-} \frac{k_{1}^{\perp} n^{-} \delta\left(p^{-}-k_{1}^{-}\right)}{k_{1}^{+}\left(k_{1}^{-}-\frac{k_{1}^{\perp 2}}{k_{1}^{+}}+\frac{i \varepsilon}{k_{1}^{+}}\right)} \\
& \times\left[\frac{k_{1}^{-}-k_{1 \text { on }}^{-}}{k_{1}^{+}\left(k_{1}^{-}-k_{1 \text { on }}^{-}+\frac{i \varepsilon}{k_{1}^{+}}\right)}\right]_{\mathrm{SS}}
\end{aligned}
$$

The result is:

$$
S^{(1) \perp-}\left(p^{-}\right)=\frac{\theta\left(p^{+}\right) p^{\perp} n^{-}}{p^{+}}\left[\frac{p^{-}-p_{\mathrm{on}}^{-}}{p^{+}\left(p^{-}-p_{\mathrm{on}}^{-}+\frac{i \varepsilon}{p^{+}}\right)}\right]_{\mathrm{SS}} \frac{i}{\left(p^{-}-K_{0}^{(1)-}+i \varepsilon\right)},
$$

where we have introduced the definition

$$
K_{0}^{(1)-}=p_{\text {on }}^{-}=\frac{p^{\perp 2}}{p^{+}},
$$

However, since the Dirac delta funtion $\delta\left(p^{-}-k_{1}^{-}\right)$forces us onto the mass-shell, the numerator is identically zero, that is,

$$
p^{-}-p_{\text {on }}^{-}=0
$$

and this is true for massless as well as massive gauge bosons.

Thus, finally

$$
S^{(1) \perp-}\left(p^{-}\right)=0 .
$$

For the component $S^{--}$we have:

$$
S^{(1)--}\left(p^{-}\right)=i \int d k_{1}^{-} \frac{k_{1}^{\perp 2} n^{-} n^{-} \delta\left(p^{-}-k_{1}^{-}\right)}{k_{1}^{+}\left(k_{1}^{-}-\frac{k_{1}^{\perp 2}}{k_{1}^{+}}+\frac{i \varepsilon}{k_{1}^{+}}\right)}\left[\frac{1}{\left(k_{1}^{+}\right)^{2}}\right]_{\mathrm{SS}}
$$




$$
\begin{gathered}
=i \int d k_{1}^{-} \frac{k_{1}^{\perp 2} n^{-} n^{-} \delta\left(p^{-}-k_{1}^{-}\right)}{k_{1}^{+}\left(k_{1}^{-}-\frac{k_{1}^{\perp 2}}{k_{1}^{+}}+\frac{i \varepsilon}{k_{1}^{+}}\right)} \\
\times\left[\frac{k_{1}^{-}-k_{1 \text { on }}^{-}}{k_{1}^{+}\left(k_{1}^{-}-k_{1 \mathrm{on}}^{-}+\frac{i \varepsilon}{k_{1}^{+}}\right)}\right]_{\mathrm{SS}}^{2}
\end{gathered}
$$

which results in:

$$
S^{(1)--}\left(p^{-}\right)=\frac{\theta\left(p^{+}\right) p^{\perp 2} n^{-} n^{-}}{p^{+}}\left[\frac{p^{-}-p_{\mathrm{on}}^{-}}{p^{+}\left(p^{-}-p_{\mathrm{on}}^{-}+\frac{i \varepsilon}{p^{+}}\right)}\right]_{\mathrm{SS}}^{2} \frac{i}{\left(p^{-}-K_{0}^{(1)-}+i \varepsilon\right)} .
$$

For the same reason as before stated,

$$
S^{(1)--}\left(p^{-}\right)=0
$$

In the case of $S^{(1) \perp \perp}\left(p^{-}\right)$component we have

$$
S^{(1) \perp \perp}\left(P^{-}\right)=\frac{\theta\left(p^{+}\right)}{p^{+}} \frac{i\left(-g^{\perp \perp}\right)}{\left(p^{-}-K_{0}^{(1)-}+i \varepsilon\right)}
$$

Clearly, this case does not present us with the $p^{+}=0$ difficulty, and the only non-vanishing result is just $S^{(1) \perp \perp}$. Only the physical degrees of freedom (transverse ones) do propagate and without zero mode hindrances anywhere!

\section{THE TWO GAUGE BOSONS CASE}

Let us go component by component in the possible non-vanishing contributuions as given in (29). For $S^{(2) \perp-, \perp-}$ we have

$$
\begin{aligned}
S^{(2) \perp-, \perp-}\left(P^{-}\right) & =-\frac{1}{(2 \pi)} \int \frac{d k_{1}^{-}}{k_{1}^{+}\left(P^{+}-k_{1}^{+}\right)} \\
& \times\left[\frac{k_{1}^{-}-k_{1 \mathrm{on}}^{-}}{k_{1}^{+}\left(k_{1}^{-}-k_{1 \mathrm{on}}^{-}+\frac{i \varepsilon}{k_{1}^{+}}\right)}\right]_{\mathrm{SS}} \frac{n^{-} k_{1}^{\perp}}{\left(k_{1}^{-}-\frac{k_{1}^{\perp}}{k_{1}^{+}}+\frac{i \varepsilon}{k_{1}^{+}}\right)} \\
& \times\left[\frac{P^{-}-k_{1}^{-}-k_{2 \mathrm{on}}^{-}}{\left(P^{+}-k_{1}^{+}\right)\left(P^{-}-k_{1}^{-}-k_{2 \mathrm{on}}^{-}+\frac{i \varepsilon}{P^{+}-k_{1}^{+}}\right)}\right]_{\mathrm{SS}} \\
& \times \frac{n^{-} k_{2}^{\perp}}{\left(P^{-}-k_{1}^{-}-\frac{\left(P^{\perp}-k_{1}^{\perp}\right)^{2}}{P^{+}-k_{1}^{+}}+\frac{i \varepsilon}{P^{+}-k_{1}^{+}}\right)} .
\end{aligned}
$$

Evaluating the residue at the pole

$$
k_{1}^{-}=k_{1 \mathrm{on}}^{-}-\frac{i \varepsilon}{k_{1}^{+}}
$$

we have

$$
S^{(2) \perp-, \perp-}\left(P^{-}\right)=0
$$

For the $S^{(2)--,--}$-component we have

$$
S^{(2)--,--}\left(P^{-}\right)=-\frac{1}{(2 \pi)} \int \frac{d k_{1}^{-}}{k_{1}^{+}\left(P^{+}-k_{1}^{+}\right)}
$$




$$
\begin{aligned}
& \times\left[\frac{k_{1}^{-}-k_{1 \mathrm{on}}^{-}}{k_{1}^{+}\left(k_{1}^{-}-k_{1 \mathrm{on}}^{-}+\frac{i \varepsilon}{k_{1}^{+}}\right)}\right]_{\mathrm{SS}}^{2} \frac{k_{1}^{\perp 2} n^{-} n^{-}}{\left(k_{1}^{-}-\frac{k_{1}^{\perp 2}}{k_{1}^{+}}+\frac{i \varepsilon}{k_{1}^{+}}\right)} \\
& \times\left[\frac{P^{-}-k_{1}^{-}-k_{2 \mathrm{on}}^{-}}{\left(P^{+}-k_{1}^{+}\right)\left(P^{-}-k_{1}^{-}-k_{2 \mathrm{on}}^{-}+\frac{i \varepsilon}{P^{+}-k_{1}^{+}}\right)}\right]_{\mathrm{SS}}^{2} \\
& \times \frac{k_{2}^{\perp 2} n^{-} n^{-}}{\left(P^{-}-k_{1}^{-}-\frac{\left(P^{\perp}-k_{1}^{\perp}\right)^{2}}{P^{+}-k_{1}^{+}}+\frac{i \varepsilon}{P^{+}-k_{1}^{+}}\right)} .
\end{aligned}
$$

yielding also

$$
S^{(2)--,--}\left(P^{-}\right)=0
$$

For the component $S^{(2) \perp-,--}$ we have

$$
\begin{aligned}
S^{(2) \perp-,--}\left(P^{-}\right) & =-\frac{1}{(2 \pi)} \int \frac{d k_{1}^{-}}{k_{1}^{+}\left(P^{+}-k_{1}^{+}\right)} \\
& \times\left[\frac{k_{1}^{-}-k_{1 \mathrm{on}}^{-}}{k_{1}^{+}\left(k_{1}^{-}-k_{1 \mathrm{on}}^{-}+\frac{i \varepsilon}{k_{1}^{+}}\right)}\right]_{\mathrm{SS}} \frac{k_{1}^{\perp} n^{-}}{\left(k_{1}^{-}-\frac{k_{1}^{\perp}}{k_{1}^{+}}+\frac{i \varepsilon}{k_{1}^{+}}\right)} \\
& \times\left[\frac{P^{-}-k_{1}^{-}-k_{2 \mathrm{on}}^{-}}{\left(P^{+}-k_{1}^{+}\right)\left(P^{-}-k_{1}^{-}-k_{2 \mathrm{on}}^{-}+\frac{i \varepsilon}{P^{+}-k_{1}^{+}}\right)}\right]_{\mathrm{SS}}^{2} \\
& \times \frac{k_{2}^{\perp 2} n^{-} n^{-}}{\left(P^{-}-k_{1}^{-}-\frac{\left(P^{\perp}-k_{1}^{\perp}\right)^{2}}{P^{+}-k_{1}^{+}}+\frac{i \varepsilon}{P^{+}-k_{1}^{+}}\right)} .
\end{aligned}
$$

which results in

$$
S^{(2) \perp-,--}\left(P^{-}\right)=0
$$

For the component $S^{(2) \perp-, \perp \perp}$ we have

$$
\begin{aligned}
S^{(2) \perp-, \perp \perp}\left(P^{-}\right) & =-\frac{1}{(2 \pi)} \int \frac{d k_{1}^{-}}{k_{1}^{+}\left(P^{+}-k_{1}^{+}\right)} \\
& \times\left[\frac{k_{1}^{-}-k_{1 \mathrm{on}}^{-}}{k_{1}^{+}\left(k_{1}^{-}-k_{1 \mathrm{on}}^{-}+\frac{i \varepsilon}{k_{1}^{+}}\right)}\right]_{\mathrm{SS}} \frac{k_{1}^{\perp} n^{-}}{\left(k_{1}^{-}-\frac{k_{1}^{\perp}}{k_{1}^{+}}+\frac{i \varepsilon}{k_{1}^{+}}\right)} \\
& \times \frac{\left(-g^{\perp \perp}\right)}{\left(P^{-}-k_{1}^{-}-\frac{\left(P^{\perp}-k_{1}^{\perp}\right)^{2}}{P^{+}-k_{1}^{+}}+\frac{i \varepsilon}{P^{+}-k_{1}^{+}}\right)} .
\end{aligned}
$$

leading us to he same vanishing result

$$
S^{(2) \perp-, \perp \perp}\left(P^{-}\right)=0
$$

The component $S^{(2)--, \perp \perp}$ is given by

$$
\begin{aligned}
S^{(2)--, \perp \perp}\left(P^{-}\right) & =-\frac{1}{(2 \pi)} \int \frac{d k_{1}^{-}}{k_{1}^{+}\left(P^{+}-k_{1}^{+}\right)} \\
& \times\left[\frac{k_{1}^{-}-k_{1 \mathrm{on}}^{-}}{k_{1}^{+}\left(k_{1}^{-}-k_{1 \mathrm{on}}^{-}+\frac{i \varepsilon}{k_{1}^{+}}\right)}\right]_{\mathrm{SS}}^{2} \frac{k_{1}^{\perp 2} n^{-} n^{-}}{\left(k_{1}^{-}-\frac{k_{1}^{\perp}}{k_{1}^{+}}+\frac{i \varepsilon}{k_{1}^{+}}\right)} \\
& \times \frac{\left(-g^{\perp \perp}\right)}{\left(P^{-}-k_{1}^{-}-\frac{\left(P^{\perp}-k_{1}^{\perp}\right)^{2}}{P^{+}-k_{1}^{+}}+\frac{i \varepsilon}{P^{+}-k_{1}^{+}}\right)} .
\end{aligned}
$$


which also yields

$$
S^{(2)--, \perp \perp}\left(P^{-}\right)=0
$$

Finally, for the component $S^{(2) \perp \perp, \perp \perp}$ we have

$$
\begin{aligned}
S^{(2) \perp \perp, \perp \perp}\left(P^{-}\right) & =-\frac{1}{(2 \pi)} \int \frac{d k_{1}^{-}}{k_{1}^{+}\left(P^{+}-k_{1}^{+}\right)} \frac{\left(-g^{\perp \perp}\right)}{\left(k_{1}^{-}-\frac{k_{1}^{\perp 2}}{k_{1}^{+}}+\frac{i \varepsilon}{k_{1}^{+}}\right)} \\
& \times \frac{\left(-g^{\perp \perp}\right)}{\left(P^{-}-k_{1}^{-}-\frac{\left(P^{\perp}-k_{1}^{\perp}\right)^{2}}{P^{+}-k_{1}^{+}}+\frac{i \varepsilon}{P^{+}-k_{1}^{+}}\right)} .
\end{aligned}
$$

which yields the non-vanishing contribution

$$
S^{(2) \perp \perp, \perp \perp}\left(P^{-}\right)=\frac{\theta\left(k_{1}^{+}\right) \theta\left(P^{+}-k_{1}^{+}\right)}{k_{1}^{+}\left(P^{+}-k_{1}^{+}\right)} \frac{i\left(-g^{\perp \perp}\right)\left(-g^{\perp \perp}\right)}{\left(P^{-}-K_{0}^{(2)-}+i \varepsilon\right)},
$$

which is the same as that obtained through the ML-prescription, (32).

Therefore, as long as we treat the troublesome zero modes $k^{+}=0$ via the singularity-softening prescription (34) the only non-vanishing component of the two gauge boson propagator is the $(\perp, \perp)$-component, so that there is no zero mode problem left and the only propagating modes are the physical, transverse ones!

\section{CONCLUSION}

Projecting the Feynman covariant space propagator into the light-front coordinates and using the MandelstamLeibbrandt prescription to treat the $k^{+}=0$ singularities we got propagation of one and two bodies in the light-front. However, the result is beset with zero-mode pathology, for the one gauge boson case and also for the two boson case in non-vanishing components such as $(\perp-, \perp-)$; the only exception being the $(\perp \perp, \perp \perp)$-component where there is no singularity of this type. We observe that even with the use of the Leibbrandt-Mandelstam prescription, it was not possible to remove the built-in singularity in $k^{+}=0$ - the outstanding zero mode problem of light-front.

Now, with the introduction of a causality preserving and singularity-softening prescription, we were able to remove the zero mode problem and let only the physical degrees of freedom propagate, namely, the transverse ones, not only in one gauge boson propagation but also in the propagation of two gauge bosons.

Acknowledgments: A.T.S. wishes to thank the kind hospitality of Physics Department, North Carolina State University, Raleigh, NC and acknowledges research grant in the earlier part of this work from CNPq (Brasília, DF), superseded by a grant from CAPES (Brasília, DF). J.H.O.S. thanks for the hospitality of Instituto de Física TeóricaUNESP, which provided facilities for the completion of this work.

[1] P.A.M.Dirac, Rev.Mod.Phys.21, 392 (1949).

[2] A.T. Suzuki and J.H.O.Sales, Nucl.Phys. A725 (2003) 139.

[3] A.T.Suzuki and A.G.M.Schmidt, Prog.Theor.Phys.103 (2000) 1011.

[4] J.B.Kogur and D.E.Soper, Phys.Rev.D1, 2901 (1970); E.Tomboulis, Phys. Rev.D8 2736 (1973); J.M.Cornwall, Phys.Rev.D10 500 (1974); P.P.Srivastava and S.J.Brodsky, Phys.Rev.D64 045006 (2001).

[5] S.Mandelstam, Nucl.Phys. B213, 149 (1983). G. Leibbrandt, Phys. Rev. D29, 1699 (1984)

[6] B.M.Pimentel and A.T.Suzuki, Phys.Rev.D42, 2115 (1990).

[7] A.T.Suzuki, Mod.Phys.Lett. A8 2365 (1993) 